\title{
Reduction of medium voltage distribution network losses through the reactive power generation in PV systems
}

\author{
N. Srećković ${ }^{1}$, E. Belič ${ }^{1}$ K. Dežan ${ }^{1}$, M. Rošer ${ }^{2}$, M. Trbušić1 ${ }^{1}$ and Gorazd Štumberger ${ }^{1}$ \\ ${ }^{1}$ University of Maribor \\ Faculty of Electrical Engineering and Computer Science \\ Smetanova 17, 2000 Maribor, Slovenia \\ Phone: +3862220 7056, Fax: +38622525481 \\ e-mail: nevena.sreckovic@um.si, ernest.belic@um.si, katarina.dezan@um.si, mislav.trbusic@um.si, \\ gorazd.stumberger@um.si \\ ${ }^{2}$ Elektro Celje d. d., \\ Vrunčeva 2a, 3000 Celje \\ Phone: +386 34201000 , fax +38634201010 \\ e-mail: miran.roser@elektro-celje.si
}

\begin{abstract}
With inevitable increase in numbers of distributed generation units connected within the distribution network (DN), different aspects of their impact on DN operation must be evaluated. This paper deals with the problem of reactive power generation from photovoltaic (PV) systems, connected to the DN, and technical and economic benefits that can be achieved on DN operation with its optimal generation. Detailed consideration of four existing medium voltage DNs with their time-dependent loading and PV generation profiles, allowed the evaluation of annual losses of electric energy, for different scenarios of reactive power generation. Furthermore, the resulting financial savings were assessed as well. Results show that with reactive power generation from installed PV units, meeting the actual needs of the network, losses can be significantly reduced and financial savings achieved.
\end{abstract}

\section{Key words}

Distribution network, PV systems, reactive power, minimization of losses

\section{Introduction}

Numerous political and environmental directives are driving the proliferation in numbers of renewable energy sources connected within the distribution networks (DN), changing them from once a passive, to an active part of a power network. If these sources are not properly placed and sized within the DN, voltage control, power quality, protection systems, fault levels and network losses may be affected [1]. Nevertheless, if they are properly incorporated within the network, numerous technical and economic benefits can be achieved on the DN operation [2], [3]. For example, if reactive power from photovoltaic (PV) systems is generated where unnecessary, reverse power flows, increase of voltages and power losses occur. On the other hand, optimal reactive power generation, meeting the needs of the network, improves the voltage profiles and reduces the active power losses [4]. Power and energy losses represent the considerable share of active power transported from transmission network to final consumers. Therefore, various approaches of control of reactive power generated from PV systems are increasingly being used as a mean to reduce these losses within the DN [5],[6].

This paper analyzes the reactive power generation from photovoltaic (PV) systems, connected to the DN, and benefits that can be achieved on DN operation with its generation, optimized for every operating state considered. Section 2 describes the evaluation of annual losses and the procedure for their minimization using the optimization method - differential evolution. Four existing medium voltage (MV) networks, presented in Section 3, with time-dependent loading and PV generation profiles were considered in the analysis. The results present the annual reduction of energy losses and financial savings obtained through proper generation of reactive power. The comparison of reduction of losses is given in Section 4 for two different scenarios of optimal reactive power generation. Considered were scenario where reactive power generation was equally distributed among the all PV systems, and the scenario where reactive power generation was determined for every PV system individually, in a way that suits the DN operation the most. Section 5 gives the final remarks and concludes the paper.

\section{Losses in distribution network.}

\section{A. Annual losses of electrical energy}

Series of time-discrete load flow calculations were performed in order to calculate daily, and hence annual energy losses. For these purposes, average time-discrete 
power loading and generation profiles were created, based on the long-term measurements of the power consumed, and power generated from PV systems within the discussed networks. Average day of every month is therefore represented with both the loading and PV generation profiles, composed of 24 discrete points given in one-hour intervals. These profiles, given in per-unit system are presented in Figure 1 and Figure 2.

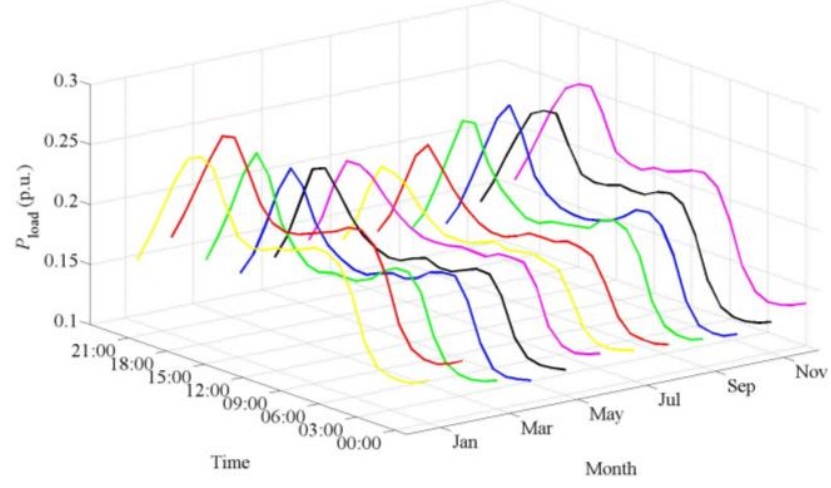

Figure 1: Loading profiles for average days in a month.

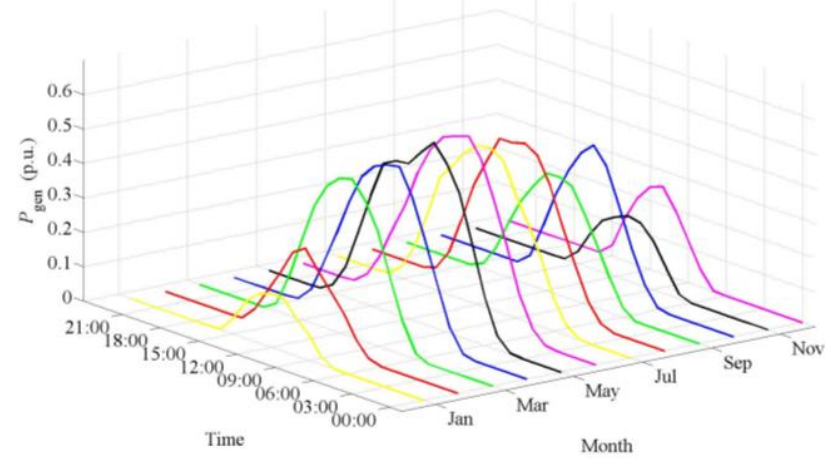

Figure 2: PV generation for average day in a month.

For every individual point in time (hour $h$ in a month $m$ ), load flow calculation was performed, resulting in calculated active power losses for the considered time point $P_{\text {loss }_{\mathrm{m}, \mathrm{h}}}$. These losses are composed of active power losses in the line segments, and losses in iron and copper of $110 / 20 \mathrm{kV}$ substation and 20/0.4 kV distribution substations, and are result of an electrical current flowing through these elements. Repeating the load flow calculation for every hour of average day in month (therefore, performing 288 time discrete load flow calculations) and knowing the number of days in every month $N_{\text {days }}$, annual losses of electrical energy within the network $W_{\text {loss }}$ are determined using (1).

$$
W_{\text {loss }}=\sum_{m=1}^{12}\left(N_{\text {days }_{\mathrm{m}}} \sum_{h=1}^{24} P_{\text {loss }_{\mathrm{m}, \mathrm{h}}}\right)
$$

Load flow calculation method used is "Backward-Forward Sweep" method [7], although other fast and robust methods, suitable for radial networks, could be used as well. Only the medium voltage part of the DN (20 kV feeder) was considered in this paper and since the unbalanced nature of $\mathrm{DN}$ is reduced with higher voltage levels, a single-phase consideration was sufficient for the analysis.

\section{B. Optimal generation of reactive power}

It has already been pointed out that the reactive power generation can be adjusted to fit the actual conditions in the network. These optimal amounts of reactive power generation are in this paper determined using the optimization method - differential evolution (DE) [8], performed for every time point with the sunlight available. DE mimics evolution in nature, including the processes of mutation, crossover, and selection, in order to create a diverse population of possible search parameters. This way, the results that satisfy the objective of the chosen fitness function $q_{\text {fun }}$ can be found. Objective in this paper is to find those amounts of reactive power that result in minimal losses within the DN $W_{\text {loss_oPT. Therefore, the }}$ fitness function is as given in (2).

$$
\min q_{\text {fun }}=\frac{W_{\text {loss_oPT }}}{W_{\text {loss }}}+p
$$

Penalties $p$ are used to introduce different calculation restrictions, including that voltages must be constrained within the limits required by the network operators $( \pm 10 \%)$, as well as to guarantee that current limits aren't exceeded in any line segment.

Minimization of annual losses of electrical energy is perform for two different scenarios of reactive power generation:

1) SCENARIO 1: reactive power generation is equally distributed among the all PV systems, proportionally to their rated powers;

2) SCENARIO 2: reactive power generation is determined for every PV system individually, in a way that suits the DN operation the most.

In both scenarios, generation was constrained with $\cos \varphi \geq 0.8$ and reactive power having capacitive nature, resulting in slight increase of voltage profiles but reduction of power losses.

\section{Test sites}

Reduction of losses of electrical energy within the DN, achieved on annual level due to the generation of reactive power from PV systems for given scenarios, was performed on four existing medium voltage distribution networks, representing four $20 \mathrm{kV}$ feeders from two $110 / 20 \mathrm{kV}$ substations. Chosen networks are mostly rural and are composed of overhead Al-Fe alloy line segments. Information about their configuration: number of line segments, nodes, 20/0.4 kV distribution substations, and connected DG units is given in Table 1.

\begin{tabular}{|c|c|c|c|c|c|c|c|}
\hline \multirow[b]{2}{*}{ DN model } & \multirow{2}{*}{$\begin{array}{l}110 / 20 \mathrm{kV} \\
\text { Substation }\end{array}$} & \multirow{2}{*}{$\begin{array}{l}20 \mathrm{kV} \\
\text { feeder }\end{array}$} & \multirow{2}{*}{\begin{tabular}{|c}
$\begin{array}{c}20 / 0.4 \mathrm{kV} \\
\text { Substations }\end{array}$ \\
Number
\end{tabular}} & \multicolumn{2}{|c|}{ DG units } & \multirow[b]{2}{*}{ Nodes } & \multirow[b]{2}{*}{ Lines } \\
\hline & & & & Number & $\begin{array}{c}\text { Rated power } \\
(\mathrm{MWp})\end{array}$ & & \\
\hline MV DN 1 & \multirow{2}{*}{ Breg } & Breg & 27 & 6 & 1.23 & 89 & 88 \\
\hline MV DN 2 & & Majšperk & 61 & 14 & 3.82 & 190 & 189 \\
\hline MV DN 3 & \multirow{2}{*}{$\begin{array}{l}\text { Rogaška } \\
\text { Slatina }\end{array}$} & Podčetrtek & 52 & 18 & 1.97 & 209 & 208 \\
\hline MV DN 4 & & Zibika & 48 & 7 & 0.84 & 196 & 195 \\
\hline
\end{tabular}

Table 1: Information about test sites. 

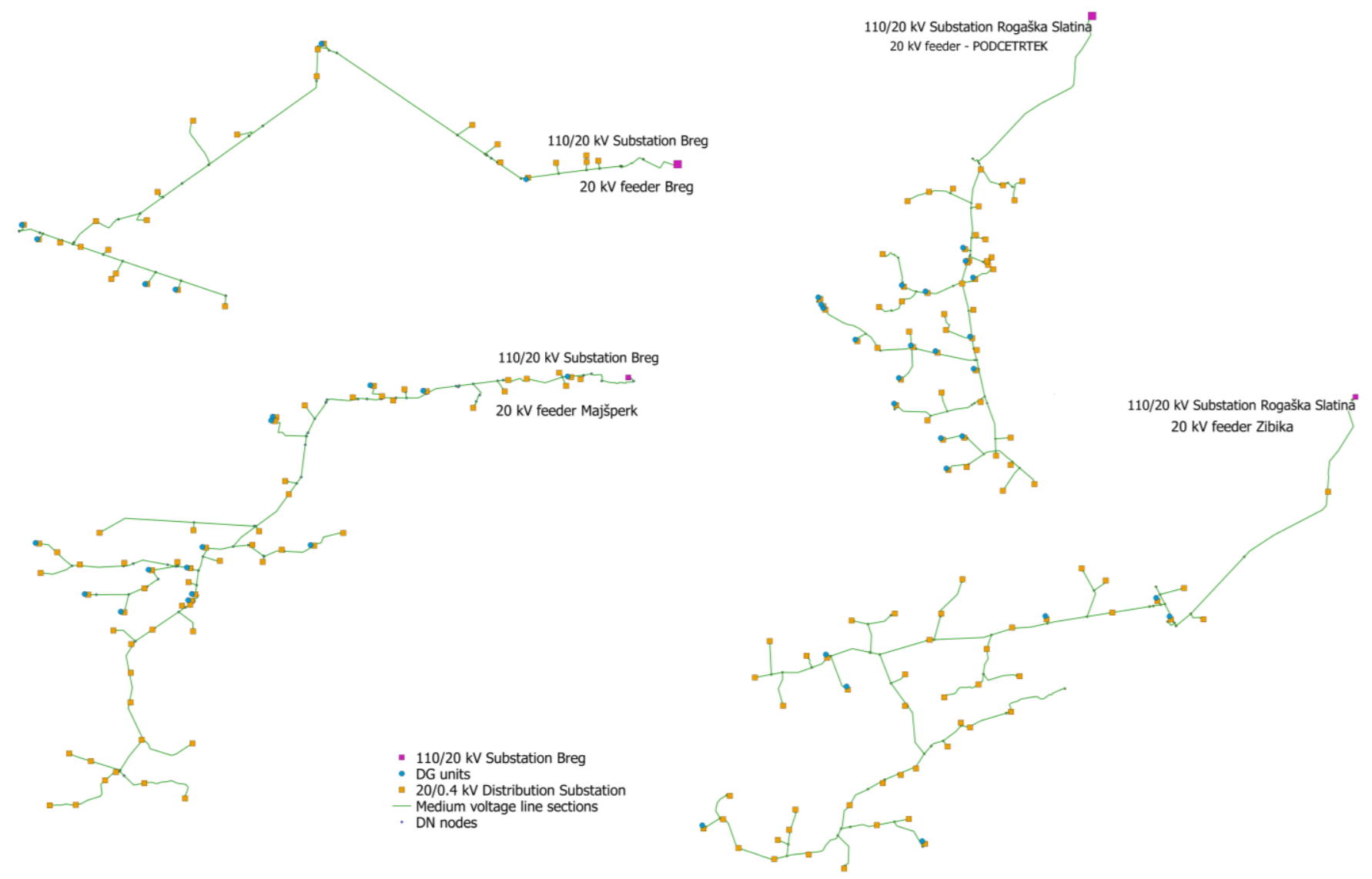

Figure 3: Single line diagrams of test medium voltage DN.

Single line diagrams of the discussed networks are given in Figure 3.

\section{Results}

For medium voltage DNs with time-dependent loading and generation profiles given in Section 2 and Section 3, minimization of losses of electrical energy was performed for scenarios 1 and 2 regarding distribution of reactive power generation among the PV systems in each network. Furthermore, using the price for the losses of electrical energy $44.45 € / \mathrm{MWh}$, financial savings that are achieved in a year, due to the reduction of losses - caused by the optimal reactive power generation are calculated.

Final results for test sites' annual financial savings and savings in electrical energy are given in Table 2. Results show that proper generation of reactive power can result in minimization of losses in DN by up to $16 \mathrm{MWh}$ (case of MV DN 2). Scenario 2 gave better results for two test DN (MV DN 2 and MV DN 3), since the reactive power generation was locally adjusted to the needs of the specific parts of the network. For test MV DN 1 and MV DN 4, results were the same as in scenario 1. The reason for this is the fact, that in both cases, all the reactive power available was generated, and it didn't matter if the optimization was performed for each PV system separately. However, it should be mentioned that the values of savings obtain are in direct dependency with the network loading and power generation profiles, and different values would be obtained for different operating conditions.
Table 2: Annual financial savings and savings in electrical energy for four test medium voltage DN.

\begin{tabular}{|c|c|c|c|c|}
\hline & & $\begin{array}{c}W_{\text {loss }}-W_{\text {loss_OPT }} \\
{[\mathrm{MWh}]}\end{array}$ & $\begin{array}{c}\text { Price } \\
{[€ / \mathrm{kWh}]}\end{array}$ & $\begin{array}{c}\text { Savings } \\
{[€]}\end{array}$ \\
\hline $\bar{z}_{0}$ & SCENARIO 1 & 5.0136 & & 222.47 \\
\hline$\sum \infty$ & SCENARIO 2 & 5.0136 & & 222.47 \\
\hline 굼 & SCENARIO 1 & 16.0152 & & 711.91 \\
\hline$\sum \sum$ & SCENARIO 2 & 16.2883 & & 723.91 \\
\hline$m \frac{y}{\underline{y}}$ & SCENARIO 1 & 5.9257 & 44.45 & 263.19 \\
\hline$\sum 0$ & SCENARIO 2 & 6.2001 & & 276.12 \\
\hline 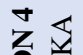 & SCENARIO 1 & 3.0416 & & 138.06 \\
\hline$\sum \nabla$ & SCENARIO 2 & 3.0416 & & 138.06 \\
\hline
\end{tabular}

Figure 4 shows savings in electrical energy for every hour $h$ of a specific day in a month $m$ for test site MV DN 3, obtained using SCENARIO 1. It can be observed that the savings occur during the daytime, and are highest around the noon in the late spring and summer months. 


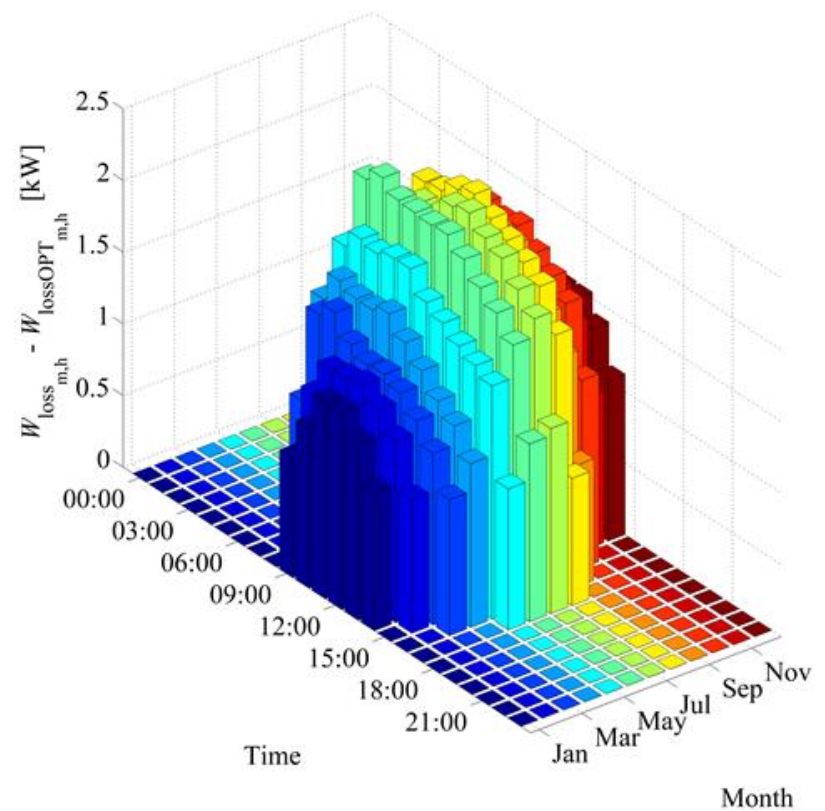

Figure 4: Savings in electrical energy for every hour of a specific day in a month (MV DN 3).

By observing the change of losses in elements of the DN, presented in Table 3, it can be seen that the greatest reduction of losses of electrical energy in the medium voltage distribution networks are achieved in distribution line segments. Change of losses in substations is almost negligible, since currents flowing through these elements are much smaller than currents in line segments.

Table 3: Annual savings of electrical energy in different elements of test MV DN.

\begin{tabular}{|c|c|c|c|c|c|}
\hline & \multicolumn{4}{|c|}{$W_{\text {loss }}-W_{\text {loss_OPT }}[\mathrm{kWh}]$} \\
\hline & & $\begin{array}{l}\text { whole } \\
\text { system }\end{array}$ & $\begin{array}{c}\text { line } \\
\text { segments }\end{array}$ & $\begin{array}{c}20 / 0.4 \\
\text { distribution } \\
\text { substations }\end{array}$ & $\begin{array}{c}110 / 20 \\
\text { substation }\end{array}$ \\
\hline \multirow{2}{*}{ 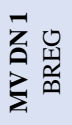 } & SCENARIO 1 & 5013.6 & 5014.0 & -0.641 & 0.242 \\
\hline & SCENARIO 2 & 5013.6 & 5014.0 & -0.641 & 0.242 \\
\hline \multirow{2}{*}{ 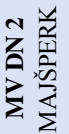 } & SCENARIO 1 & 16015.2 & 16102.3 & -87.700 & 0.688 \\
\hline & SCENARIO 2 & 16288.3 & 16327.0 & -37.399 & 0.682 \\
\hline \multirow{2}{*}{ 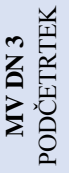 } & SCENARIO 1 & 5925.7 & 5924.1 & 0.876 & 0.739 \\
\hline & SCENARIO 2 & 6200.1 & 6198.7 & 0.581 & 0.737 \\
\hline \multirow{2}{*}{ 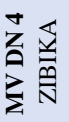 } & SCENARIO 1 & 3041.6 & 3041.3 & -0.065 & 0.430 \\
\hline & SCENARIO 2 & 3041.6 & 3041.3 & -0.065 & 0.430 \\
\hline
\end{tabular}

\section{Conclusion}

The purpose of this paper was to investigate the technical and economic benefits of the reactive power generation, adjusted to the actual needs of the four existing medium voltage DNs. The results of the analysis, presented in Section 4 have shown that noteworthy financial savings and savings in electrical energy can be achieved with optimal reactive power generation. Since reactive power generated had capacitive nature, power losses were reduced, but on the other hand, voltage profiles increased. However, the use of penalties in the optimization procedure guaranteed that voltage profiles are constrained within the limits, required by the distribution system operators. Reduction of losses of electrical energy occurs mostly in the power lines, while change in losses in $110 / 20 \mathrm{kV}$ and $20 / 0.4 \mathrm{kV}$ distribution substations is almost negligible, due to small current values flowing through substations. Savings achieved are higher if the generation is optimized for every PV system individually. However, optimization performed this way is more computationally demanding, since there were more parameters that had to be determined.

Furthermore, it is important to mention that generation of reactive power from PV systems is not completely loss free. It has been shown, that the efficiency of microinverters reduces with the generation of reactive power, yet this reduction is merely up to $2 \%$ in the considered operating states, and was therefore neglected in this analysis. Further research will include the efficiency characteristics of microinverters, allowing reduction of active power generation to be determined as well.

\section{Acknowledgement}

This work was supported in part by Slovenian Research Agency under research contract L2-5489.

\section{References}

[1] J. Driesen and R. Belmans, "Distributed generation: challenges and possible solutions," in IEEE Power Engineering Society General Meeting, 2006, 2006, p. 8 pp.-.

[2] E. J. Coster, J. M. A. Myrzik, B. Kruimer, and W. L. Kling, "Integration Issues of Distributed Generation in Distribution Grids," Proc. IEEE, vol. 99, no. 1, pp. 28-39, Jan. 2011

[3] T. Stetz, M. Kraiczy, M. Braun, and S. Schmidt, "Technical and economical assessment of voltage control strategies in distribution grids," Prog. Photovolt. Res. Appl., vol. 21, no. 6, pp. 1292-1307, Sep. 2013.

[4] V. H. M. Quezada, J. R. Abbad, and T. G. S. Román, "Assessment of energy distribution losses for increasing penetration of distributed generation," IEEE Trans. Power Syst., vol. 21, no. 2, pp. 533-540, May 2006.

[5] S. Bolognani, R. Carli, G. Cavraro, and S. Zampieri, "Distributed Reactive Power Feedback Control for Voltage Regulation and Loss Minimization," IEEE Trans. Autom. Control, vol. 60, no. 4, pp. 966-981, Apr. 2015.

[6] A. Cagnano, E. De Tuglie, M. Liserre, and R. A. Mastromauro, "Online Optimal Reactive Power Control Strategy of PV Inverters," IEEE Transactions on Industrial Electronics, vol. 58, no. 10, pp. 4549-4558, Oct. 2011.

[7] D. Thukaram, H. M. Wijekoon Banda, and J. Jerome, "A robust three phase power flow algorithm for radial distribution systems," Electr. Power Syst. Res., vol. 50, no. 3, pp. 227-236, Jun. 1999.

[8] R. Storn and K. Price, "Differential Evolution - A Simple and Efficient Heuristic for global Optimization over Continuous Spaces," J. Glob. Optim., vol. 11, no. 4, pp. 341-359, Dec. 1997. 